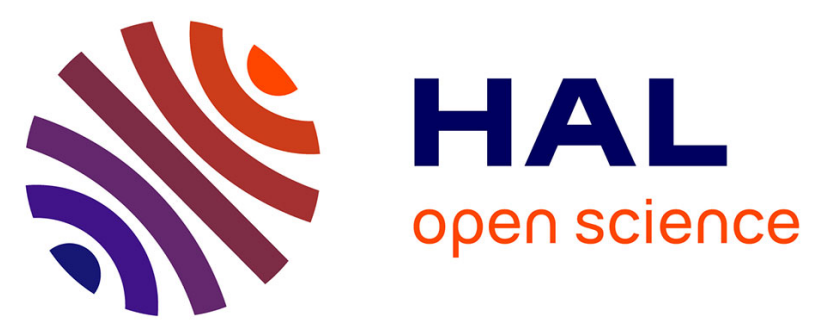

\title{
Synthesis and Application of FRET Nanoparticles in the Profiling of a Protease
}

Lionel Marcon, Corentin Spriet, Timothy D Meehan, Bronwyn J Battersby, Gwendolyn A Lawrie, Laurent Héliot, Matt Trau

\section{- To cite this version:}

Lionel Marcon, Corentin Spriet, Timothy D Meehan, Bronwyn J Battersby, Gwendolyn A Lawrie, et al.. Synthesis and Application of FRET Nanoparticles in the Profiling of a Protease. Small, 2009, 5 (18), pp.2053 - 2056. 10.1002/smll.200801887 . hal-03025747

\section{HAL Id: hal-03025747 https://hal.science/hal-03025747}

Submitted on 14 Oct 2021

HAL is a multi-disciplinary open access archive for the deposit and dissemination of scientific research documents, whether they are published or not. The documents may come from teaching and research institutions in France or abroad, or from public or private research centers.
L'archive ouverte pluridisciplinaire HAL, est destinée au dépôt et à la diffusion de documents scientifiques de niveau recherche, publiés ou non, émanant des établissements d'enseignement et de recherche français ou étrangers, des laboratoires publics ou privés. 


\section{Combinatorial libraries}

L. Marcon, C. Spriet, * T. D. Meehan, B. J. Battersby, G. A. Lawrie, L. Héliot, M. Trau 1-5

Synthesis and Application of FRET Nanoparticles in the Profiling of a Protease
Fluorescent silica nanoparticles incorporating unique ratios of energy transfer dyes are synthesized and applied as colloidal barcodes to encode a microsphere-bound combinatorial peptide library. The affinity of the West Nile virus protease is profiled using this library with cleavage of the peptide detected by flow cytometry. The cleaved peptide substrates are sorted and then identified through decoding by confocal microscopy combined with spectral unmixing (see image). 


\title{
Synthesis and Application of FRET Nanoparticles in the Profiling of a Protease*
}

\author{
Lionel Marcon, Corentin Spriet, ${ }^{\star}$ Timothy D. Meehan, Bronwyn J. Battersby, \\ Gwendolyn A. Lawrie, Laurent Héliot, and Matt Trau
}

Disease biomarker identification, pathogen detection, drug discovery, and biomolecular profiling require assays utilizing large numbers of molecules. ${ }^{[1-3]}$ These assays typically involve screening chemical libraries to identify target molecules such as antigens, nucleotides, or peptides. Split-and-mix synthesis has emerged as a powerful strategy for creating enormous combinatorial libraries, in which multiple products are synthesized simultaneously on solid support microspheres. ${ }^{[4]}$ The conventional strategy for encoding split-and-mix libraries involves covalent binding of chemical "identifier" tags (e.g., nucleic acids, secondary amines, fluorophenyl ethers $)^{[5]}$ to the microspheres in parallel with the compound synthesis. However, there are many advantages associated with the use of optical encoding, rather than chemical encoding, with the major advantage being the ability to use flow cytometry for rapid biomolecular analysis.

Several attempts have been made to combine fluorescence encoding methods with solid phase library synthesis. ${ }^{[6-9]}$ These methods generally use "pre-encoded" support microspheres, that is, the supports are encoded before the library synthesis proceeds. However, we recently introduced a method for optically encoding solid phase libraries in parallel with, rather than prior to, combinatorial synthesis (i.e., colloidal barcoding). ${ }^{[10-14]}$ This unique method involves encoding solid support

[*] Dr. C. Spriet, Dr. L. Héliot

Biophotonique cellulaire fonctionnelle

Institut de Recherche Interdisciplinaire

Université des Sciences et Technologies de Lille

USR 3078 CNRS, Parc de la Haute Borne, 50 avenue de Halley, 59658 Villeneuve d'Ascq (France)

E-mail: Corentin.Spriet@iri.univ-lille1.fr

Dr. L. Marcon, Dr. T. D. Meehan, Dr. B. J. Battersby, Dr. G. A. Lawrie, Prof. M. Trau

Centre for Nanotechnology and Biomaterials

Australian Institute for Bioengineering and Nanotechnology

University of Queensland, St. Lucia, QLD 4072 (Australia)

[**] Recombinant WNV protease was kindly donated by Dr. Paul Young (School of Molecular and Microbial Sciences, University of Queensland, Australia). The authors acknowledge Damien Schapman and the Biophotonic Core Facility of Lille (USR3078/IRI). This work was supported by the ARC (FF0455861; DP0555914) and the NHMRC (301267). We acknowledge the Centre for Microscopy and Microanalysis (UQ) for the use of electron microscopy equipment. The authors L. Marcon and C. Spriet had an equal contribution.

․ Supporting Information is available on the WwW under http:// www.small-journal.com or from the author.

DOI: $10.1002 / \mathrm{smll} .200801887$ microspheres $(10-20 \mu \mathrm{m})$ with smaller silica reporter particles $(0.1-0.8 \mu \mathrm{m})$ in order to tag each step during library synthesis. The reporters contain a single dye, or combinations of dyes, which impart unique spectral signatures that can be decoded by 4 fluorescence microscopy to identify the compounds synthe- 5 sized. ${ }^{[10,11]}$ Colloidal barcoding has been successfully utilized to 6 produce a library of 100 encoded tripeptides ${ }^{[10]}$ with the 7 possibility of encoding a library containing 64 million chemical 8 species by incorporating combinations of only seven dyes into 9 the reporters.

After conducting a biomolecular assay with the solid phase library, the support microspheres that bear bioactive library members are sorted rapidly via a flow cytometer. Conventional fluorescence microscopy can be used to manually read the colloidal barcode on each support microsphere and requires differentiation of up to seven different dyes incorporated within the reporters that form the colloidal barcode. This necessitates the use of multiple filters to limit the overlap in the excitation and emission spectra of the reporters. An alternative strategy is described here and involves incorporating different combinations of energy transfer fluorescent dyes within the silica reporters. By varying the concentrations of dyes, the reporters can exhibit multiple colors with tunable emission signatures by fluorescence resonance energy transfer (FRET) under a single wavelength excitation. FRET only occurs when the emission and absorption spectra of the donor and acceptor overlap and when the molecules are separated by a critical distance $(1-10 \mathrm{~nm})$. This is achieved in the reporter particles due to the covalent localization of the fluorophores within a silica matrix. This strategy has been demonstrated using polystyrene microspheres ${ }^{[15]}$ and silica particles ${ }^{[16]}$ in other studies, however, has not before been applied to optical encoding in combinatorial chemistry. The traditional filter-based decoding approach lacks the resolution required to decode the complex spectral signatures produced by FRET reporters, ${ }^{[17]}$ however confocal microscopy can be used to quantify the contribution of different dyes in each reporter. ${ }^{[18]}$

In this paper, we present a novel colloidal barcoding method utilizing nanoscale FRET reporters decoded by confocal laser scanning microscopy combined with spectral unmixing. This strategy was implemented to encode a combinatorial peptide library that was directed against a model protease (Figure 1). Fluorescent silica reporters were synthesized through a modified Stöber procedure that has been described in detail elsewhere. ${ }^{[19]}$ Briefly, succinimidyl ester dyes Atto-488 $\left(\lambda_{\mathrm{ex}} 501 / \lambda_{\mathrm{em}} 523 \mathrm{~nm}\right)$, Atto-550 $\left(\lambda_{\mathrm{ex}} 554 / \lambda_{\mathrm{em}} 576 \mathrm{~nm}\right)$, and 46 
a)

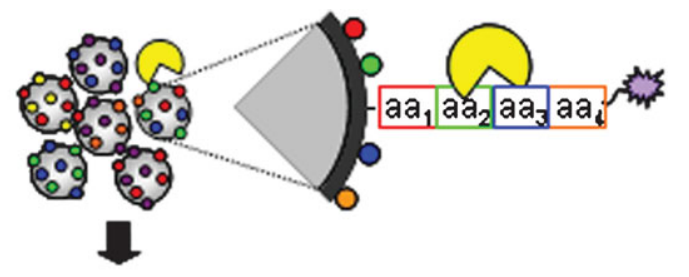

b)
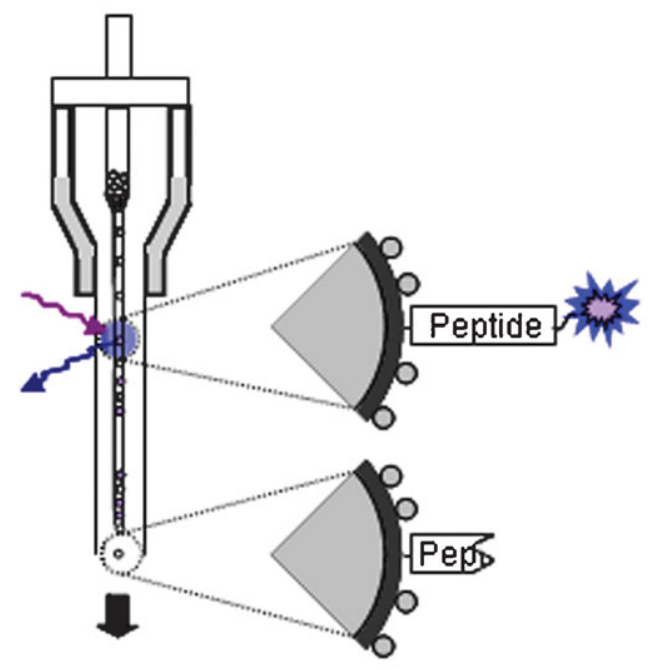

c)

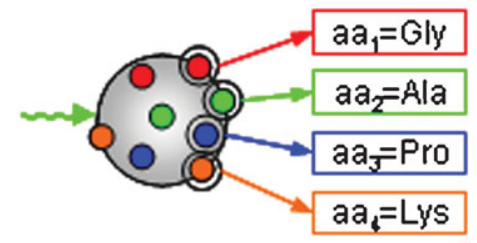

Figure 1. Principle of the colloidal barcoding method. a) Optically barcoded combinatorial peptide libraries synthesized on support microspheres and terminated with a dye (violet) are incubated with a protease (yellow). For clarity, aa stands for amino acid. b) The fluorescence of the terminal dye is detected by flow cytometry. Microspheres exhibiting a loss of the terminal dye are sorted. c) The hit microspheres are then decoded by confocal microscopy to identify the sequence of the attached peptide.
Atto-590 $\left(\lambda_{\mathrm{ex}} 594 / \lambda_{\mathrm{em}} 624 \mathrm{~nm}\right)$ (Atto-Tec, Siegen, Germany) were covalently linked to a silane coupling agent (3-aminopropyl)trimethoxysilane (APS, Aldrich, USA) forming an APS-dye conjugate. These three dyes were selected to enable FRET using a single $488 \mathrm{~nm}$ excitation source resulting from the spectral overlap between the donor's emission and the acceptor's excitation (Figure 2a). Silica particle formation was initiated by reacting the APS-dye conjugate with tetraethoxysilane (TEOS, Aldrich) and ammonia $\left(\mathrm{NH}_{4} \mathrm{OH}\right)$. The resulting reporters were coated with a polyelectrolyte bilayer composed of poly(diallyldimethylammonium chloride) and poly(acrylic acid) (Aldrich) to promote their adhesion to the support microspheres. ${ }^{[10,11]}$

As a first step in the synthesis of the encoded library, it was necessary to tune the synthesis of the reporters to optimize the size and homogeneity of the particles. This was crucial to the success of the encoding strategy as the reporters must both i) permit the incorporation of a sufficient concentration of dyes to be distinguishable by confocal microscopy and ii) be
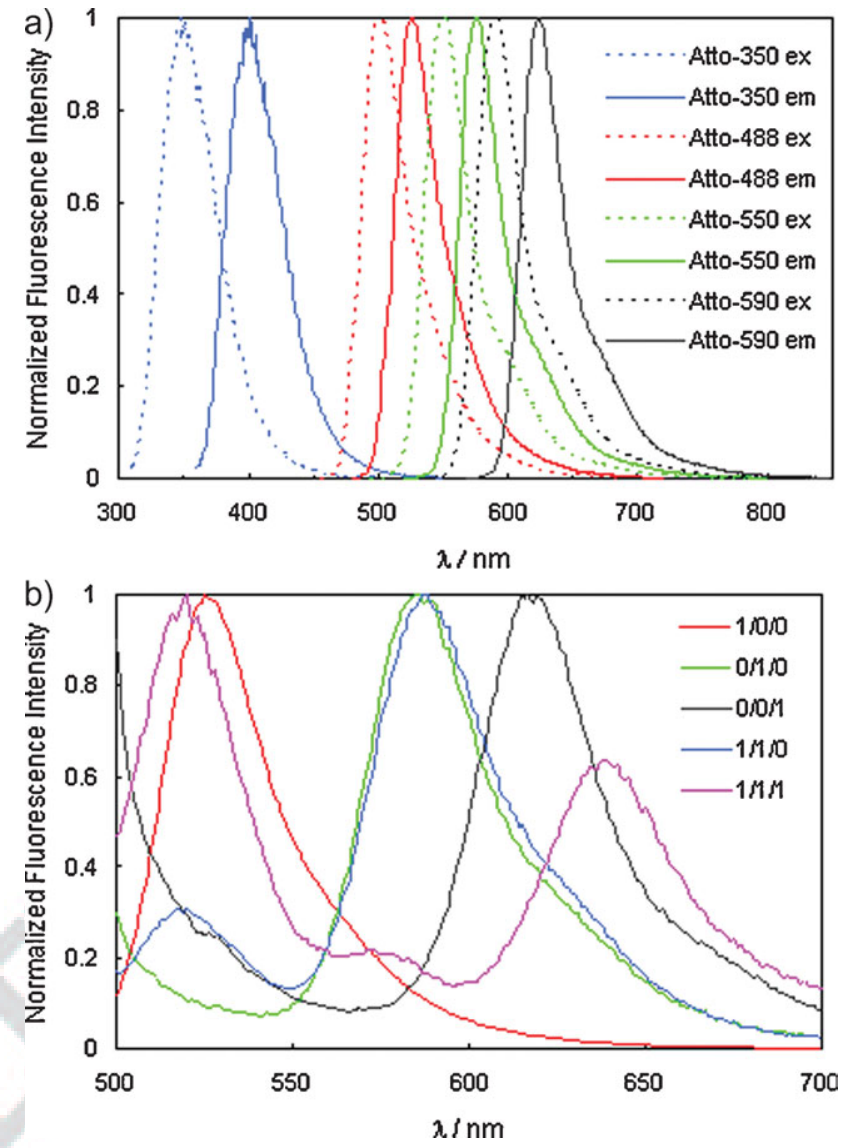

Figure 2. a) Fluorescence spectra (provided by the supplier) of the dyes used in this study. Dashed lines and continuous lines represent the excitation and emission spectra respectively. b) Spectrally unique emission signatures of reporters measured for $1 \times$ series.

sufficiently small to maximize the number of reporters that could be attached to the support microspheres. Previous studies, indicating that the particle size and dispersity were functions of the concentration of $\mathrm{NH}_{4} \mathrm{OH}$ used in the synthesis, were used as a guide in this investigation. ${ }^{[20,21]}$ Concentrations from 0.8 to $6.3 \mathrm{M}$ resulted in reporter sizes ranging from $50 \pm 3 \mathrm{~nm}$ to $850 \pm 15 \mathrm{~nm}$, respectively (see Supporting Information). A secondary nucleation was observed at concentrations greater than $4.2 \mathrm{~m}$ that resulted in an undesirable polydisperse particle size distribution and the formation of aggregates. Considering the initial decoding observations by confocal microscopy (data not shown), it was determined that the $120 \pm 5 \mathrm{~nm}$ reporters synthesized using $4.2 \mathrm{M} \mathrm{NH} \mathrm{NH}_{4} \mathrm{OH}$ represented the best combination of high fluorescence signal and narrow size distribution.

To obtain distinguishable emission signatures, the three dyes were incorporated into the reporters at five molar ratios $(0,1 \times, 2 \times, 3 \times$, and $5 \times)$. For example, a reporter having $1 \times$ of Atto- $488,2 \times$ of Atto-550, and no Atto-590 dye (code 1/2/0) was distinguishable from a reporter having $1 \times$ of Atto- $488,2 \times$ of Atto-550, and $1 \times$ of Atto-590 dye (1/2/1). To illustrate FRET between the three dyes within the reporters and that the signatures were distinguishable, the fluorescence emission spectra of the ratio $1 \times$ reporter series are presented in 
Figure 2b. It is apparent, by comparing the spectra of the double $(1 / 1 / 0)$ and triple-dyed reporters (1/1/1) with those of the singledyed reporters $(1 / 0 / 0,0 / 1 / 0$, and $0 / 0 / 1)$, that the emission of the donor Atto-488 dramatically decreased when associated with Atto-550 and/or Atto-590. Inversely, the emission of the acceptor moieties was shown to increase. A red-shift in emission, as noted in a previous study, was also observed here when the dyes were combined. ${ }^{[15]}$

To evaluate the utility of the reporters to form a colloidal barcode, the reporters were loaded on $10 \mu \mathrm{m}$ amine functionalized polystyrene microspheres (Tentagel, Rapp Polymere, Tübingen, Germany) in eleven different combinations of between three and seven codes (see composition in Supporting Information). Reference spectra for the three dyes alone were acquired from the single-dyed reporters (1/0/0,0/1/0, and 0/0/1) using a Leica SP2 confocal microscope (Leica Microsystems, Wetzlar, Germany). Utilizing these reference spectra as a calibration, the contributions of each dye to the composite fluorescence signature of multiple-dyed reporters were quantified using the spectral unmixing algorithm supplied with the acquisition LCS software.

The adhesion of the reporter particles to a microsphere is shown in the scanning electron microscopy (SEM) image (JEOL JSM 6400F, Tokyo, Japan) in Figure 3a and an example of the decoding of a microsphere bearing a combination of 4 reporters $(5 / 1 / 1,1 / 5 / 0,1 / 1 / 5$, and $5 / 1 / 0)$ is presented in Figure $3 b-d$. Analysis of the spectra of the four reporters presented in Figure $3 \mathrm{~d}$ demonstrates that they are readily distinguishable. The spectral reproducibility of reporters attached to microspheres was assessed and found to have an average coefficient of variation equal to $5 \%$. Decoding data (image and fluorescence spectra) of the most significant combinations are given in the Supporting Information along with the experimental set-up.

Following characterization, the reporters were used to encode a combinatorial peptide library that was applied to profiling the affinity of the West Nile flavivirus protease (WNV-pro). WNV is a potentially lethal human pathogen found throughout the tropical world. The protease produced by the virus is required to cleave the precursor viral polyprotein into the individual functional proteins that are essential for viral replication. ${ }^{[22]}$ Previous studies by Chappell et al. and Shiryaev et al. defined the optimal WNV-pro substrates as Leu-Gly-X-X $\downarrow$ Gly-Gly-Ala-Ala where $\mathrm{X}$ is either Arg or Lys and the cleavage site is represented by $\downarrow .^{[23,24]}$ These researchers demonstrated that the proteolysis was most profoundly affected by an alteration of the $\mathrm{X}-\mathrm{X}$ dibasic motif.

On this basis, a library composed of 144 octapeptides was constructed via a split-and-mix method using natural amino acids by standard Fmoc chemistry in which the $\mathrm{X}-\mathrm{X}$ position was encoded. The amino acids in the $\mathrm{X}-\mathrm{X}$ positions were randomized from a pool of twelve amino acids to incorporate a representative of each significant category (acidic, basic, hydrophilic, and hydrophobic). The library and its encoding are described in the Supporting Information. Following the peptide synthesis, Atto-350 ( $\left.\lambda_{\mathrm{ex}} 350 / \lambda_{\mathrm{em}} 423 \mathrm{~nm}\right)$, a dye excited in the UV range and with an emission spectrum different from those of the reporters, was covalently linked to the terminal amino acid of each peptide (Figure 4a).

The library was incubated with the WNV-pro for $1 \mathrm{~h}$ at $37^{\circ} \mathrm{C}$. Proteolysis was detected via flow cytometry (BD LSRII, Becton Dickinson, North Ryde, Australia) through the reduction of on-microsphere fluorescence of Atto-350
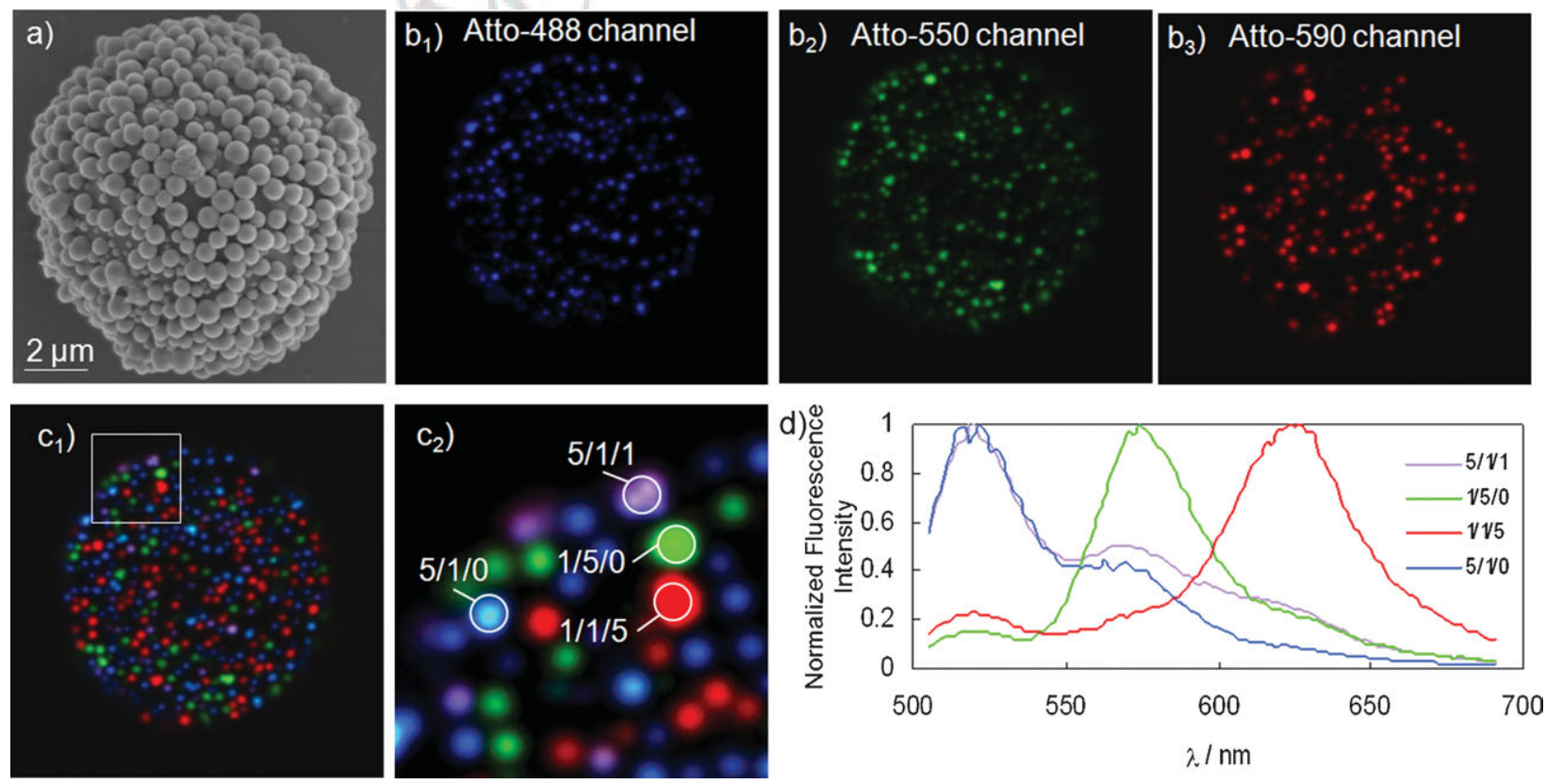

Figure 3. a) SEM image of a $10 \mu \mathrm{m}$ microsphere with $120 \mathrm{~nm}$ silica reporters. $\mathrm{b}_{1-3}$ ) Contribution of each dye to the total fluorescence of the reporters after spectral unmixing. $c_{1,2}$ ) Composite image merging the three contributions and d) identification of each reporter's specific fluorescence code. These confocal images are represented using false colors for visual contrast. 
a)

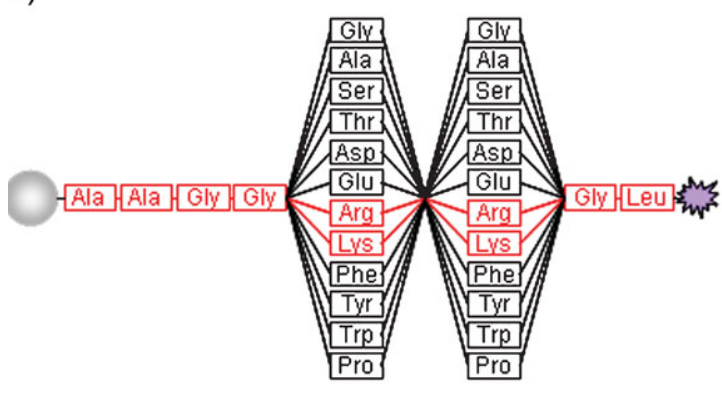

b)

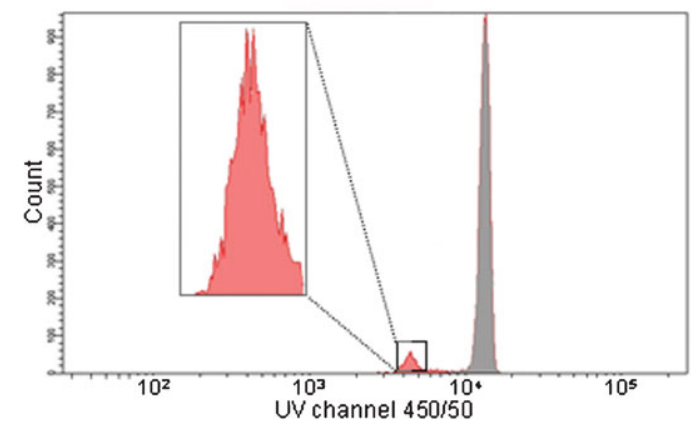

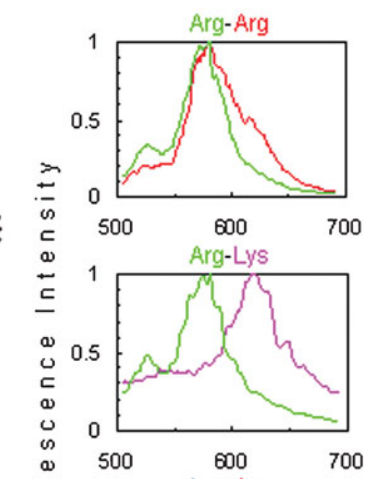

c)

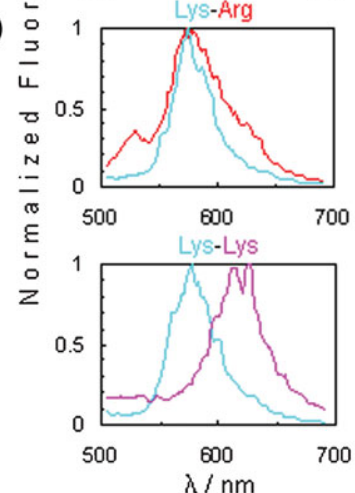

Figure 4. a) Microsphere-bound peptide library produced by split-and-mix synthesis. Sequences in red lettering represent the substrates that were identified after decoding. b) Flow cytometry analysis after incubation of the peptide library with the WNV-pro. The population exhibiting a fluorescence decrease (shown in red) was collected for decoding. c) Corresponding barcodes.

(Figure 4b). A population exhibiting a fluorescence decrease of $80 \%$, indicating proteolytic cleavage, was sorted and collected for confocal decoding. Four sequences, containing Arg and Lys in the two randomized positions, were identified in this population (indicated by red lettering in Figure 4a), in agreement with those substrates identified in the solutionbased studies previously discussed. ${ }^{[23,24]}$ Representative composite spectra of the reporters encoding these randomized positions of the four sequences are presented in Figure 4c.

By utilizing $d$ number of dyes at $r$ number of ratios, $\left(r^{d}-1\right)$ unique optical codes can be generated. ${ }^{[25]}$ The use here of three dyes $(d=3)$ incorporated at five ratios $(r=5)$ therefore has the potential to generate a total of 124 unique codes according to $\left(5^{3}-1\right)$. If one considers the creation of an encoded hexapeptide library in which every amino acid was randomized from a pool of all 20 natural amino acids, it can be calculated that only 120 codes would be required to distinguish all $\left(20^{6}\right) 64$ million unique peptides. Protease screening routinely utilizes peptides of no more than six amino acids in length. ${ }^{[26]}$ Therefore, our methodology can be directly applied to the profiling of newly discovered proteases with unknown affinities. However, encoding other chemical libraries (nucleotides, carbohydrates, etc.) might necessitate a greater number of FRET reporters. This could be accomplished by correlating emission spectra acquisitions with dye lifetimes. The direct determination of fluorescence lifetime is indeed one of the most direct measures of FRET. ${ }^{[27]}$ In addition, the use of multiple excitations would extend the useful spectral range of the reporters enabling the creation of larger encoded libraries.

In summary, we reported here for the first time the synthesis of a combinatorial peptide library encoded using FRET reporter nanoparticles. Varying the ratios of energy transfer dyes served as an innovative route to produce unique spectral signatures. In addition, the use of confocal microscopy combined with spectral unmixing represents a major improvement in the interrogation of optically barcoded libraries by enabling the recognition of complex dye combinations. Our profiling of the West Nile virus protease was consistent with previous studies done with standard methods and represents an important step toward the practical implementation of colloidal barcoding. Such supports should find broad applications in gene expression studies, high-throughput screening, and medical diagnostics.

\section{Keywords:}

barcoding $\cdot$ confocal microscopy $\cdot$ fluorescence $\cdot$ nanoparticles $\cdot$ protease

[1] H. M. Geysen, F. Schoenen, D. Wagner, R. Wagner, Nat. Rev. Drug Discovery 2003, 2, 222.

[2] O. H. Aina, R. Liu, J. L. Sutcliffe, J. Marik, C. X. Pan, K. S. Lam, Mol. Pharmaceutics 2007, 4, 631.

[3] O. Schilling, C. M. Overall, Nat. Biotechnol. 2008, 26, 685.

[4] K. S. Lam, M. Lebl, V. Krchnak, Chem. Rev. 1997, 97, 411.

[5] A. W. Czarnik, Curr. Opin. Chem. Biol. 1997, 1, 60.

[6] B. J. Egner, S. Rana, H. Smith, N. Bouloc, J. G. Frey, W. S. Brocklesby, M. Bradley, Chem. Commun. 1997, 735.

[7] L. Marcon, D. Kozak, B. J. Battersby, K. J. Chappell, D. P. Fairlie, P. Young, M. Trau, Anal. Biochem. 2008, 376, 151.

[8] A. Nanthakumar, R. T. Pon, A. Mazumder, S. Yu, A. Watson, Bioconjugate Chem. 2000, 11, 282.

[9] R. H. Scott, S. Balasubramanian, Bioorg. Med. Chem. Lett. 1997, 7, 1567.

[10] B. J. Battersby, D. Bryant, W. Meutermans, D. Matthews, M. L. Smythe, M. Trau, J. Am. Chem. Soc. 2000, 122, 2138.

[11] L. Grøndahl, B. J. Battersby, D. Bryant, M. Trau, Langmuir 2000, 16, 9709.

[12] M. Trau, B. J. Battersby, Adv. Mater. 2001, 13, 975.

[13] B. J. Battersby, G. A. Lawrie, M. Trau, Drug Discovery Today 2001, 6, 19.

[14] B. J. Battersby, M. Trau, Trends Biotechnol. 2002, 20, 167.

[15] W. B. Wu, M. L. Wang, Y. M. Sun, W. Huang, Y. P. Cui, C. X. Xu, Opt. Mater. 2008, 30, 1803.

[16] L. Wang, W. Tan, Nano Lett. 2006, 6, 84.

[17] E. A. Jares-Erijman, T. M. Jovin, Nat. Biotechnol. 2003, 21, 1387.

[18] M. E. Dickinson, G. Bearman, S. Tille, R. Lansford, S. E. Fraser, Biotechniques 2001, 31, 1272.

[19] G. Lawrie, L. Grondahl, B. Battersby, I. Keen, M. Lorentzen, P. Surawski, M. Trau, Langmuir 2006, 22, 497.

[20] R. M. Ottenbrite, J. S. Wall, J. Am. Ceram. Soc. 2000, 83, 3214.

[21] L. M. Rossi, L. Shi, F. H. Quina, Z. Rosenzweig, Langmuir 2005, 21, 4277. 
[22] T. J. Chambers, R. C. Weir, A. Grakoui, D. W. McCourt, J. F. Bazan, R. J. Fletterick, C. M. Rice, Proc. Natl. Acad. Sci. USA 1990, 87, 8898.

[23] K. J. Chappell, M. J. Stoermer, D. P. Fairlie, P. R. Young, J. Biol. Chem. 2006, 281, 38448.

[24] S. A. Shiryaev, I. A. Kozlov, B. I. Ratnikov, J. W. Smith, M. Lebl, A. Y. Strongin, Biochem. J. 2007, 401, 743.

[25] M. Han, X. Gao, J. Z. Su, S. Nie, Nat. Biotechnol. 2001, 19, 631.
[26] S. L. Diamond, Curr. Opin. Chem. Biol. 2007, 11, 46.

[27] R. M. Clegg, O. Holub, C. Gohlke, Methods Enzymol. 2003, 360, 509.

Received: December 16, 2008 Revised: April 30, 2009

Published online:

\section{2}

